\title{
Incidental intestinal malrotation in an adult: Midgut volvulus
}

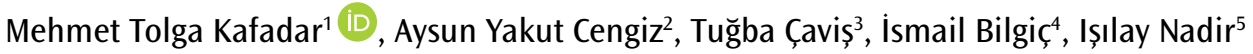

\section{ABSTRACT}

ORCID ID of the author: M.T.K. 0000-0002-9178-7843

\section{Cite this paper as:}

Kafadar MT, Cengiz AY, Caviş

T, Bilgiçi, Nadir l. Incidental intestinal malrotation in an adult: Midgut volvulus. Turk J Surg 2018; 34(4):337-339

${ }^{1}$ Clinic of General Surgery, Health Sciences University Mehmet Akif Inan Training and Research Hospital, Şanlıurfa, Turkey ${ }^{2}$ Clinic of Radiology, Medical Park Sivas Hospital, Sivas, Turkey

${ }^{3}$ Clinic of Radiology, Atatürk Training and Research Hospital, Ankara, Turkey

${ }^{4}$ Clinic of General Surgery, Ankara Umut Hospital, Ankara, Turkey

${ }^{5}$ Clinic of Gastroenterology, Medical Park Ankara Hospital, Ankara, Turkey

This study was presented at the $13^{\text {th }}$ National

Hepatogastroenterology Congress, 27 April-1 May 2016, Antalya, Turkey

\section{Corresponding Author} Mehmet Tolga Kafadar e-mail:drtolgakafadar@hotmail. com

Received: 26.11.2015 Accepted: 29.12.2015

OCopyright 2018 by Turkish Surgical Association

Available online at turkjsurg.com
Intestinal malrotation is a developmental anomaly characterized by a midgut fixation disorder and failure to complete its normal fetal rotation around the superior mesenteric artery inside the peritoneal cavity. Malrotation is a rare cause of mechanical intestinal obstruction in adults. It may manifest as an emergency condition or with chronic abdominal symptoms. Although its diagnosis remains difficult, early diagnosis and treatment usually yield favorable outcomes. Intestinal malrotation rarely has an asymptomatic course, in which the diagnosis is usually made incidentally. Multislice computed tomography is quite helpful for making a correct diagnosis. In this study, we reported a 56-year-old patient incidentally who was detected to have intestinal malrotation and was managed symptomatically after presenting at our clinic with signs and symptoms of intestinal obstruction; we also provided a discussion of the relevant literature.

Keywords: Midgut malrotation, volvulus, adult

\section{INTRODUCTION}

Midgut volvulus is a rare type of malrotation that develops as a result of the rotation of the mesenteric root around the superior mesenteric artery (1). Although it is more common in infants, it can also be encountered in adults. Midgut volvulus possible signs include the whirlpool sign in omphalomesenteric vessels, atypical location of the superior mesenteric artery and vein, and signs of intestinal malrotation. Its incidence has been reported to be one per 500 births in epidemiological studies (2). Herein, we report a 56-year-old patient with an incidental finding of intestinal malrotation presenting with signs of small intestinal obstruction.

\section{CASE PRESENTATION}

A 56-year-old man presented with the complaints of abdominal pain, nausea, vomiting, bloating, and inability to pass gas and feces for 3 days. History of patient was remarkable for intermittent abdominal pain. Hemoglobin, white blood cell count, and basic biochemistry panel were all within normal limits. He was administered an oral contrast agent to obtain a whole abdomen computed tomography (CT) scan, which showed a distended stomach filled with the orally administered contrast agent (Figure 1a). The vascular structures and mesentery twisted clockwise (whirlpool sign) at the abdominal midline, and superior mesenteric vein was located to the left of superior mesenteric artery (Figure 1b, c). No sign of necrosis was apparent in intestinal segments. The patient was hospitalized with an initial diagnosis of ileus; his oral intake was stopped and symptomatic treatment was initiated. He began to pass gas and feces 2 days after admission. Having an overall favorable general status, the patient was discharged on day 5 . He returned for a follow-up appointment 15 days later when he had no clinical problem. A Magnetic Resonance (MR) enterography was performed, which again showed the whirlpool sign (Figure 2). Informed consent was obtained from the patient who participated in this case.

\section{DISCUSSION}

Midgut volvulus is a serious anomaly that develops as a result of narrow attachment of midgut mesentery due to a rotation anomaly, resulting in clockwise twisting of intestinal segments on the superior mesenteric artery axis; it may end up with ischemia and necrosis (1). Normal rotation and fixation events in an embryo occur between the 5th and 12th weeks of gestation. Starting from the 5th week, intestinal development shifts to the interior of the umbilical cord as a result of insufficient space inside the primitive coelomic space. Ultimately, at the 10th embryonic week, intestines return to abdominal cavity and complete their rotation, with the duodenojejunal loop being in the first place. Intestinal malrotation develops as a result of a deficient rotation and fixation of the midgut segment after its passage through the umbilicus and return to coelomic space. Midgut volvulus becomes symptomatic within 1 month after birth in $80 \%$ of cases (2). 

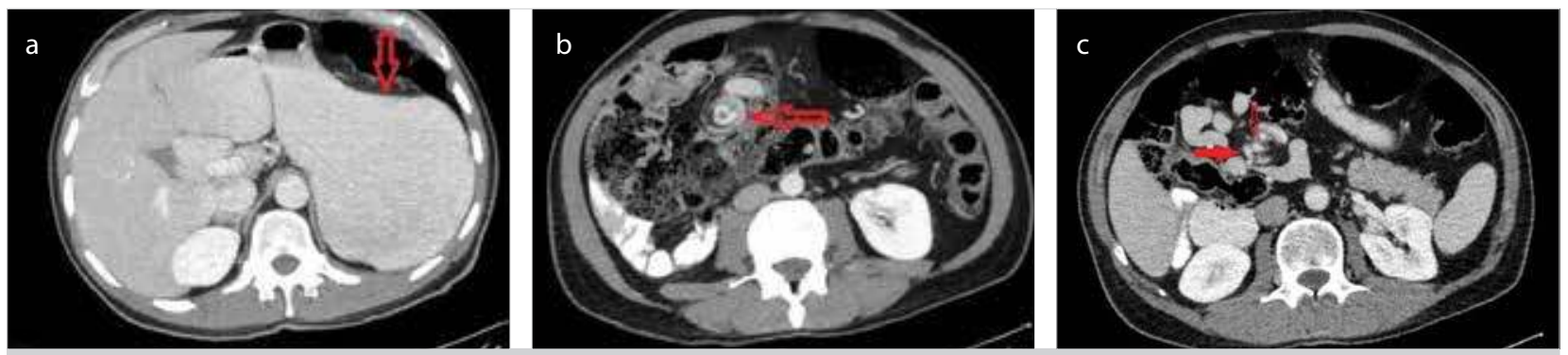

Figure 1.a-c. (a) Stomach appears distended by filling of its lumen by the orally administered contrast agent. (b) Duodenojejunal junction is located to the right of the midline; the whirlpool sign formed by the proximal jejunal segment and the surrounding mesenteric fat tissue around SMA is seen. (c) SMA (filled arrow) is visualized to the right of SMV (empty arrow)

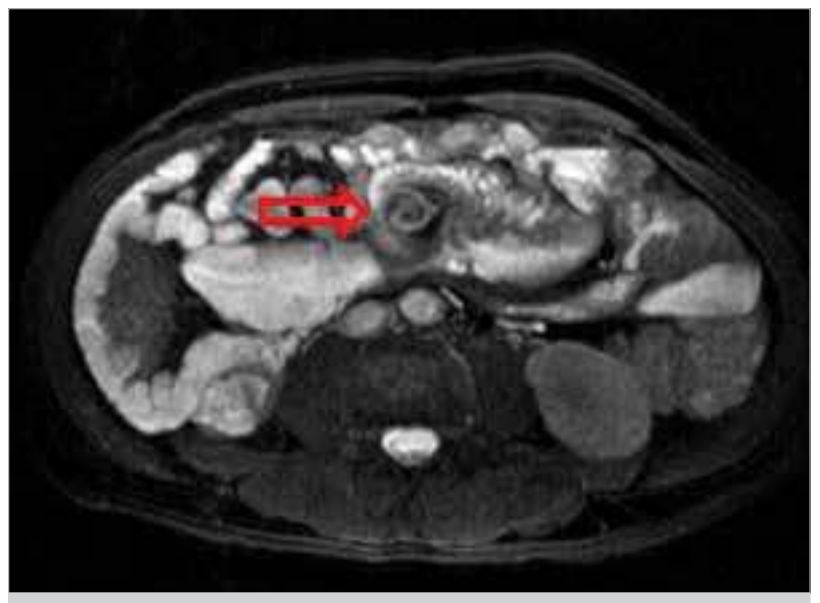

Figure 2. MR Enterography shows the typical whirlpool sign (Axial fat-suppressed T2A sequence)

Midgut volvulus is an extremely rare anomaly in adults. Patients with malrotation may be asymptomatic at an early stage. Some patients may present with chronic, unexplained abdominal discomfort or, more rarely, with acute abdominal pain. These symptoms may be secondary to Ladd's bands causing acute or chronic intestinal obstruction or volvulus (3). History, physical examination, and laboratory examinations are integral to the diagnosis of midgut volvulus. CT, ultrasonography, small intestinal passage films, and MR enterography may reveal the typical signs of midgut volvulus. Edema-induced mural thickening in the duodenum and the location of superior mesenteric vein to the left and anterior to superior mesenteric artery are diagnostic signs (4). Even in our patient, the location of the duodenojejunal junction to the right of the vertebral column, the clockwise malrotation of the mesenteric root (whirlpool sign), and the location of the vascular structures on BT were demonstrated in detail. Our case was interesting with respect to the age of the patient, rotation of mesenteric meso, and the absence of signs of ischemia.

Hanna et al. (5) reported that they initially considered diverticulitis in a patient with signs of acute abdomen due to malrotation; the authors later diagnosed midgut volvulus and a left-sided appendicitis upon the intensification of patient's symptoms, but they could not avoid a delay in diagnosis and management. Our patient also had intermittent symptoms; however, he did not care about these symptoms too much and did not seek for any medical attention owing to the mild
Although many adult patients are symptomatic, the diagnosis may still be delayed owing to the lack of specific symptoms. Many patients with malrotation may suffer incorrect diagnoses and wrong procedures due to these atypical symptoms (6). Patients may also be entirely asymptomatic. Most patients are diagnosed accidentally during an operation or at autopsy. Malrotation cases operated for ileus or acute appendicitis with left lower quadrant pain have been reported (7).

Midgut volvulus can be managed symptomatically or, sometimes, surgically. Patients with this condition are typically considered to be candidates for intestinal obstruction and are associated intestinal necrosis. Thus, some authors have recommended elective laparotomy and Ladd's procedure (6). The aim of the latter, which is the most common intervention performed in malrotation, is to cut Ladd's bands compressing the duodenum and jejunum to relieve small intestine. Other surgical procedures include duodenopexy, cecopexy, and adhesiolysis (8). Complicated volvulus may become fatal unless treated. Liao et al. (9) detected an adult patient with midgut volvulus due to delayed posttraumatic hemorrhagic mesenteric pseudocyst that was treated by urgent surgery. Önder et al. (10) on the other hand, treated a 61-year-old patient with midgut volvulus symptomatically and did not perform surgery. We also did not undertake any surgical intervention, and our patient was relieved by symptomatic treatment and was followed without any problem for 1 month.

\section{CONCLUSION}

Intestinal malrotation is a quite rare anomaly in adults, although it is highly important with respect to its potential complications. The possibility of malrotation should not be omitted in patients with non-specific abdominal complaints. Although mostly difficult, diagnosis of malrotation in adults can be possible by considering it in the differential diagnosis of small intestinal obstruction.

Informed Consent: Written informed consent was obtained from patient who participated in this study.

Peer-review: Externally peer-reviewed.

Author Contributions: Concept - M.T.K., T.Ç., I.B.; Design - M.T.K., A.Y.C.; Supervision- M.T.K., I.B.; Resource - M.T.K., I.N.; Materials - M.T.K., A.Y.C., T.Ç., I.N.; Data Collection and/or Processing - M.T.K., A.Y.C., T.Ç.; Analysis and/or Interpretation - M.T.K.; Literature Search - M.T.K., T.Ç.; Writing Manuscript - M.T.K.; Critical Reviews - M.T.K., I.B., I.N.

Conflict of Interest: The authors have no conflicts of interest to declare. 
Financial Disclosure: The authors declared that this study has received no financial support.

\section{REFERENCES}

1. Papadimitriou G, Marinis A, Papakonstantinou A. Primary midgut volvulus in adults: report of two cases and review of the literature. J Gastrointest Surg 2011; 15: 1889-1892. [CrossRef]

2. Kamal IM. Defusing the intra-abdominal ticking bomb: intestinal malrotation in children. CMAJ 2000; 162: 1315-1317.

3. Gamblin TC, Stephens RE Jr, Johnson RK, Rothwell M. Adult Malrotation: A case report and review of the literature. Curr Surg 2003; 60: 517-520. [CrossRef]

4. Wanjari AK, Deshmukh AJ, Tayde PS, Lonkar Y. Midgut malrotation with chronic abdominal pain. N Am J Med Sci 2012; 4: 196198. [CrossRef]

5. HannaT, Akoh JA. Acute presentation of intestinal malrotation in adults: a report of two cases. Ann R Coll Surg Engl 2010; 92: 15-18. [CrossRef]
6. Cohen Z, Kleiner O, Finaly R, Mordehai J. How much of a misnomer is 'asymptomatic' intestinal malrotation? Isr Med Assoc J 2003; 5: 172-174.

7. Goicochea Mancilla C, Diaz Plasencia J, Balmaceda Fraselle T. Intestinal obstruction for malrotation in an adult patient. Report of a case. Rev Gastroenterol Peru 2001; 21: 60-63.

8. Dietz DW, Walsh RM, Grundfest-Broniatowski S, Lavery IC, Fazio VW, Vogt DP. Intestinal malrotation: a rare but important cause of bowel obstruction in adults. Dis Colon Rectum 2002; 45: 13811386. [CrossRef]

9. Liao WI, LinYY, Ko KH, Chu SJ, Tsai SH. Midgut volvulus as a delayed consequence of posttraumatic mesenteric pseudocyst. Am J Emerg Med 2009; 27: 1025. [CrossRef]

10. Önder H, Özmen CA, Gümüş M, Ekici F, Bükte Y. Midgut volvulus in an adult patient due to intestinal malrotation. Sakarya Med J 2012; 2: 102-104. [CrossRef] 